\title{
Evaluation of sensation evoked by electrocutaneous stimulation on forearm in nondisabled subjects
}

\author{
Bo Geng, MSc; ${ }^{1 *}$ Ken Yoshida, PhD; ${ }^{1-2}$ Laura Petrini, PhD; ${ }^{1,3}$ Winnie Jensen, PhD $^{\mathbf{1}}$ \\ ${ }^{1}$ Department of Health Science and Technology, Aalborg University, Aalborg, Denmark; ${ }^{2}$ Biomedical Engineering \\ Department, Indiana University-Purdue University Indianapolis, Indianapolis, IN; ${ }^{3}$ Department of Communication \\ and Psychology, Aalborg University, Aalborg, Denmark
}

\begin{abstract}
Few studies are available in the literature on the sensations artificially created by dual-channel electrocutaneous stimulation. This study assessed the effect of a set of selected stimulation parameters on the sensations evoked by single- or dual-channel electrocutaneous stimulation. The investigated parameters included the stimulation site, the number of pulses, the number of stimulating channels (single- vs dual-channel), and the interleaved time between two channels. The modality, quality, location, and magnitude of the sensations were evaluated when the stimulations were applied on the forearm skin in 16 nondisabled subjects. Tactile perception was found to be induced more easily on the median and ulnar aspect than the dorsal and radial aspect of the forearm. Stimulation site significantly affected the magnitude of the sensation $(p<0.01)$. Dualchannel stimulation significantly increased the sensation magnitude $(p<0.05)$ only when the two electrodes were positioned closely. Moreover, a higher number of pulses evoked a movement perception more frequently and the interleaved time showed no significant effect on the magnitude of the sensation. The findings are expected to be useful for sensory substitution and augmentation applications. The results may also help improve users' acceptance of hand prostheses.
\end{abstract}

Key words: artificial sensation, electrocutaneous stimulation, prostheses, psychophysical test, sensation measure, sensory augmentation, sensory communication, sensory feedback, sensory modulation, sensory substitution.

\section{INTRODUCTION}

A prosthesis is an artificial device used to replace a missing body part. Today, commercially available powered hand prostheses can restore some motor functionality. However, their usefulness is limited by lack of sensory feedback. Control of a hand prosthesis is often difficult when it is moved outside the field of vision and the grip force is usually found to be insufficient [1]. Incorporating a sensory feedback system in advanced hand prostheses may overcome such difficulties and improve their usefulness [2-4].

Artificially inducing afferent nerve activity is considered a way to augment or substitute an impaired sense [5]. Electrocutaneous stimulation has been recognized as one of the feasible techniques for creating nerve activity because it is noninvasive and capable of producing sensations that can be controlled reliably [6]. A number of earlier studies reported that electrocutaneous sensory feedback improved

\footnotetext{
Abbreviations: PLP $=$ phantom limb pain, $\mathrm{PT}=$ perception threshold, VAS = visual analog scale.

*Address all correspondence to Bo Geng, MSc; Aalborg University, Center for Sensory-Motor Interaction (SMI), Department of Health Science and Technology, Fredrik Bajersvej 7D, 9220 Aalborg, Denmark; +45-9940-8798; fax: +45-9815-4008. Email: bogeng@hst.aau.dk http://dx.doi.org/10.1682/JRRD.2010.09.0187
} 
subjects' confidence in using hand prostheses and facilitated their awareness of the prostheses in their body image [7-11]. The sensory information may be encoded by modulation of stimulation parameters; for example, a subject was reported to be able to relate modulation of the pulse rate to the gripping force when using a myoelectrically controlled hand prosthesis [9].

In addition to the application of electrocutaneous stimulation in prosthetic devices, evidence has emerged that sensory feedback training may alleviate phantom limb pain (PLP) or chronic limb pain [12-13]. In a study on the effect of sensory discrimination on PLP, five amputee patients with PLP were asked to discriminate the locations and frequencies of the electrical stimulation applied to the residual limb. After 2 weeks of training, the PLP was reduced in all subjects compared with a control group that received regular PLP treatments, including analgesic medication, transcutaneous nerve stimulation, or physical therapy [12]. Also, a recent study demonstrated that tactile discrimination, rather than stimulation alone, relieved pain while improving tactile acuity in patients with complex regional pain syndrome [13]. These studies suggest that training of sensory acuity by discrimination of stimulation parameters may offer therapeutic benefit for PLP and other types of chronic pain.

In both of the applications just mentioned, an effective stimulation paradigm depends on the stimulation parameters selected for sensory modulation. Thus, investigating how stimulation parameters influence sensory output is important. A majority of earlier studies have investigated single-channel stimulation [14-16]. The use of multiple electrodes may enhance the information transfer rates by introducing additional stimulation variables [6]. However, few studies have systematically investigated the effects of stimulation parameters on evoked sensations in dual-channel electrocutaneous stimulation, which is the focus of this work.

As such, this study examined the effect of four stimulation parameters, which have previously not been extensively researched, on evoked sensation modality, magnitude, quality, and location. The investigated parameters were the stimulation site, the number of stimulating channels (singlechannel vs dual-channel), the number of pulses, and the interleaved time in dual-channel stimulation. The hypothesis was that modulation of the selected stimulation parameters could modulate evoked sensations. The evoked sensations were qualitatively and quantitatively evaluated by psychophysical tests.

\section{METHODS}

\section{Subjects}

Sixteen nondisabled human subjects (8 males and 8 females, $28.3 \pm 4.0$ years) participated in the study. All subjects signed an informed consent before the experiment. The experimental protocol was in compliance with the Declaration of Helsinki and approved by the Danish local ethics committee. The subjects had no known or visible skin diseases in the forearm and no known history of neurological or psychological disorders.

\section{Experimental Setup}

The experiments were performed on a customdesigned, computer-controlled platform. The experimental setup is shown in Figure 1. The stimulation sequences were configured in "Computer 1." The stimulus generator STG2008 (Multi Channel Systems; Reutlingen, Germany) generated an analog voltage output. The DS5 (Digitimer; Hertfordshire; United Kingdom) translated the voltage signal into an isolated current stimulus. The stimulus was delivered to one or two electrodes (or channels) selected by two manually operated switches. Immediately following a stimulus, the subject was presented with a questionnaire to describe the perceived sensation. The questionnaire was implemented in a graphical user interface displayed on "Computer 2."

Five self-adhesive Ambu (Ballerup, Denmark) Neuroline 700 solid gel electrodes (skin contact size: $20 \times 15 \mathrm{~mm}$, "duck foot" shape, silver/silver chloride) were placed around the left forearm $5 \mathrm{~cm}$ distally to the elbow joint. The five electrode sites are referred to as S1, S2, S3, S4, and S5. A schematic of the electrode placement is shown in the Figure 1 inset. The location of the five sites was standardized among the subjects according to the following rules: (1) S1 was placed over the median nerve; (2) S2 was placed laterally adjacent to S1 (center-tocenter distance: approximately $15 \mathrm{~mm}$, without any physical connection between the two electrodes); and (3) S3, S4, and S5 were equally spaced between S2 and S1. The common reference electrode was positioned over the wrist on the left forearm. We identified the location of the median nerve by applying nonpainful electrical stimulation with moderate intensity. The site of S1 was then identified as the place where the evoked sensations projected to the thenar eminence and the palmar side of the thumb, the index finger, and the middle finger. S1 and S2 were placed next to each other in order to examine the 


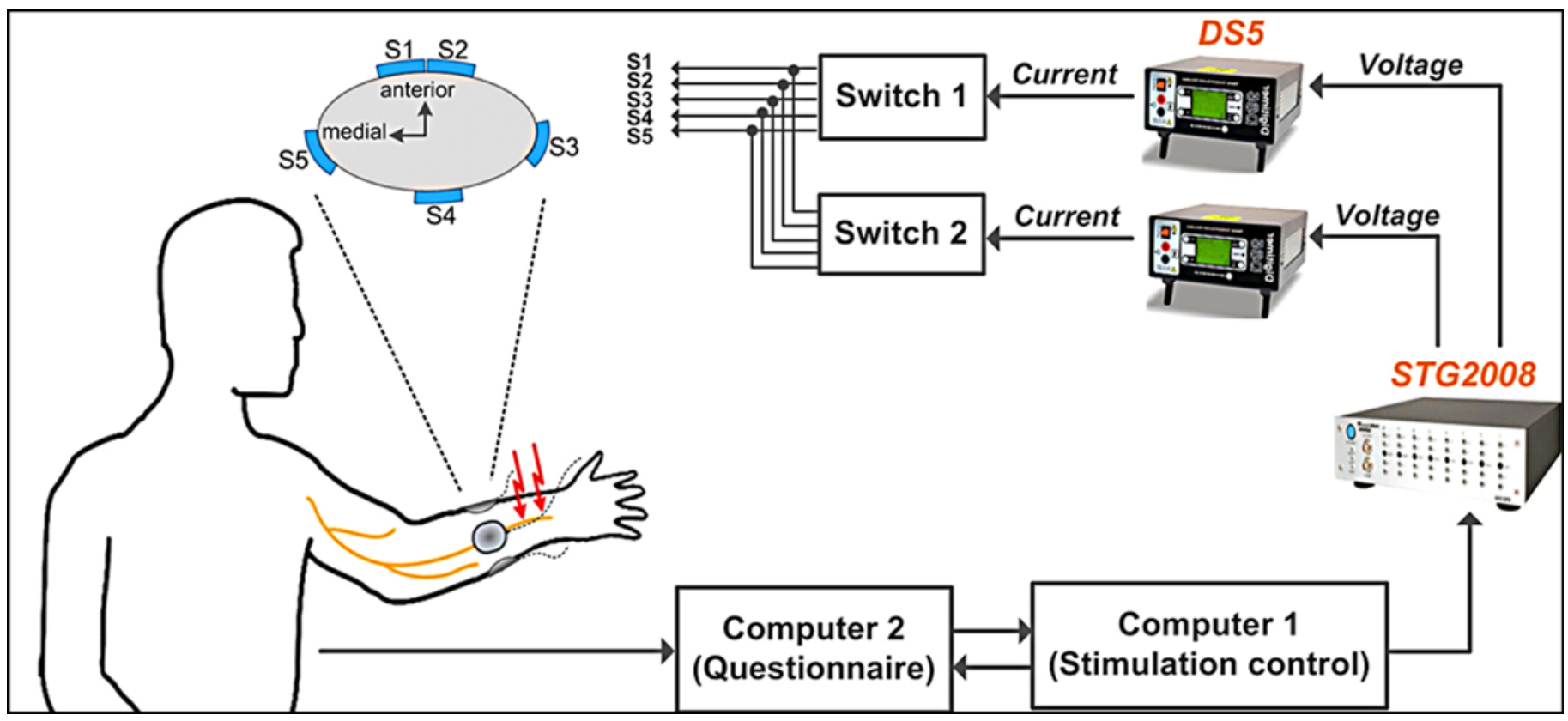

Figure 1.

Schematic of experimental setup. Stimulation control was performed in "Computer 1." STG2008 generated analog voltage output, and DS5s converted voltage into current signal. Stimuli were delivered to one or two electrodes selected by the "Switch." Subject reported perceived sensations by answering questionnaire displayed on "Computer 2." Five electrodes were placed around left forearm. Inset shows location of electrodes, where S5 is on medial side and S1 and S2 on ventral side of forearm.

effects of the distance between electrodes in dual-channel stimulation. The center-to-center distance between S3, S4, and S5 ranged from 46 to 69 mm depending on individual forearm size. We prepared the skin by gently shaving and moisturizing it with a water-soaked cotton cloth to improve conductivity.

\section{Stimulation Application}

Symmetric, rectangular, biphasic pulses at the frequency of $20 \mathrm{~Hz}$ were applied. A biphasic waveform was used since it has previously been demonstrated to produce more comfortable sensations than monophasic pulses [6]. The applied amplitude was defined as a factor of the individual subject's lowest perception threshold (PT) among five sites and was constant throughout the experiment. Since the range of the PT varied between subjects, the amplitude was chosen to be 2.5 4.0 times the lowest PT so that each subject perceived clear sensations at all sites. Pulse durations of 100, 200, and $500 \mu \mathrm{s}$ were first tested in a pilot experiment, and the pulse duration of $200 \mu$ s was selected because it produced the least uncomfortable sensation at the five electrode sites.
Four types of stimulation were applied. In type 1, a single-pulse stimulus was applied to one of the five electrodes. In type 2, two single-pulse stimuli were applied simultaneously to pairs of electrodes. Seven out of ten possible pair combinations were compared: S1\&S2, S1\&S3, S1\&S4, S1\&S5, S3\&S4, S3\&S5, and S4\&S5. The remaining three combinations-S2\&S3, S2\&S4, and S2\&S5-were not included because S1 and S2 were placed closely and the combinations with S2 led to similar results as those with S1 in the pilot experiment. In type 3, multipulse stimuli ( $n=1,2,5,10,20)$ were simultaneously applied to three pairs of electrodes (S1\&S2, S1\&S4, and S3\&S5). In type 4, two single-pulse stimuli were applied to the three pairs of electrodes with eight different values of interleaved time (i.e., $t=50 \mu \mathrm{s}, 100 \mu \mathrm{s}, 200 \mu \mathrm{s}$, $500 \mu \mathrm{s}, 1 \mathrm{~ms}, 5 \mathrm{~ms}, 10 \mathrm{~ms}, 50 \mathrm{~ms}$ ) between two channels. The three particular combinations were selected because they comprise all the five electrode sites and cover varying distances between electrodes. Throughout the experiment, the subjects were blind to the stimulation parameters. The four types of stimulation were presented to the subjects in random order, while the conditions within each stimulation type (e.g., conditions of $n=1$, 2, 
5, 10, 20 in type 3) were not randomized. The stimuli were not repeated to avoid possible adaptation. As such, a total of 48 stimuli were applied to each subject, and for each stimulus, the evoked sensation was psychophysically evaluated.

\section{Evaluation of Evoked Sensations}

Immediately after a stimulus was applied, the subject evaluated the perceived sensation with regard to the following four aspects: modality, location, quality, and magnitude. A psychophysical questionnaire consisting of three multiple-choice questions was used to measure the evoked sensation modality, location, or quality (Table). A predefined word list was provided for each question, and the subject was asked to choose the one or more words that best described the sensation. The predefined words were selected based on those words previously reported in the literature [2,6,17-18] as well as in our pilot experiments. To minimize unreliable measures, we instructed the subjects in the meaning of the words before the experiments.

The magnitude of a sensation was rated by the subject using a visual analog scale (VAS) horizontally displayed on the questionnaire. The VAS rating was as follows: 0 ("no sensation") to 10 ("upper limit of a sensation or pain"). Note that the magnitude of a sensation is a subjective experience and two subjects may rate the identical stimulus with considerably different VAS scores. Therefore, for comparison of results between subjects, the VAS scores rated by each subject were normalized by the overall maximum VAS score from the subject.

The questions were not presented in random order because this is not believed to affect the results of psychophysical tests [19]. The subject was allowed to take as much time as needed to answer the questionnaire. As such, when ready to receive the next stimulus, the subject was required to click on a button on the computer interface. The next stimulus was then delivered after approximately $5 \mathrm{~s}$.

\section{Data Analysis}

Because the results of sensation modality, location, and quality were categorical data, we used the nonparametric Cochran $Q$ test to assess whether the electrode location, the number of pulses, or the interleaved time significantly affected the evoked sensations. Because the VAS scores were ranking data, we used the Friedman test to assess whether the investigated parameters significantly affected the magnitude of the evoked sensations. We used the Wilcoxon rank sum test to compare the sensation magnitude in single-channel stimulation with that of dual-channel stimulation. The significance level was defined as 0.05 .

\section{RESULTS}

\section{Overview of Evoked Sensation Modality, Location, and Quality}

An overview of the sensation modality, location, and quality evoked by the four types of stimulation is depicted by a heat map (Figure 2). Such a heat map allows a straightforward overview of and comparison among four stimulation types.

With regard to the modality of the evoked sensations, the following observations can be made from the heat map. Overall, "touch," "vibration," "tingling," "pinprick," and "movement" were reported more frequently, whereas "cold," "warm," "pinch,” and "crush” were reported less frequently in all stimulation types. In the multiple-pulse stimulation (type 3), "tingling," "pinprick," and "movement" were mostly reported. In the dual-channel stimulation (type 2, 3, 4), stimulation of the electrode pairs involving S1 evoked "movement" more frequently and stimulation of the electrode pairs involving S3 and S4 produced "pinprick" more often.

Regarding the location of the evoked sensations, the multiple-pulse stimulation (type 3) elicited "spreading" and "referred" sensations more often and the interleaved

Table.

Questionnaire for evaluation of modality, location, and quality of evoked sensations.

\begin{tabular}{|c|c|c|}
\hline Variable & Question & Predefined Word List \\
\hline No. 1 (Modality) & What type of sensation did you feel? & $\begin{array}{l}\text { Touch, vibration, warm, cold, tingling, itch, pinprick, } \\
\text { pinch, crush, movement }\end{array}$ \\
\hline No. 3 (Quality) & How would you describe the quality of the sensation? & Comfortable, uncomfortable, sharp, blunt, mild, strong \\
\hline
\end{tabular}



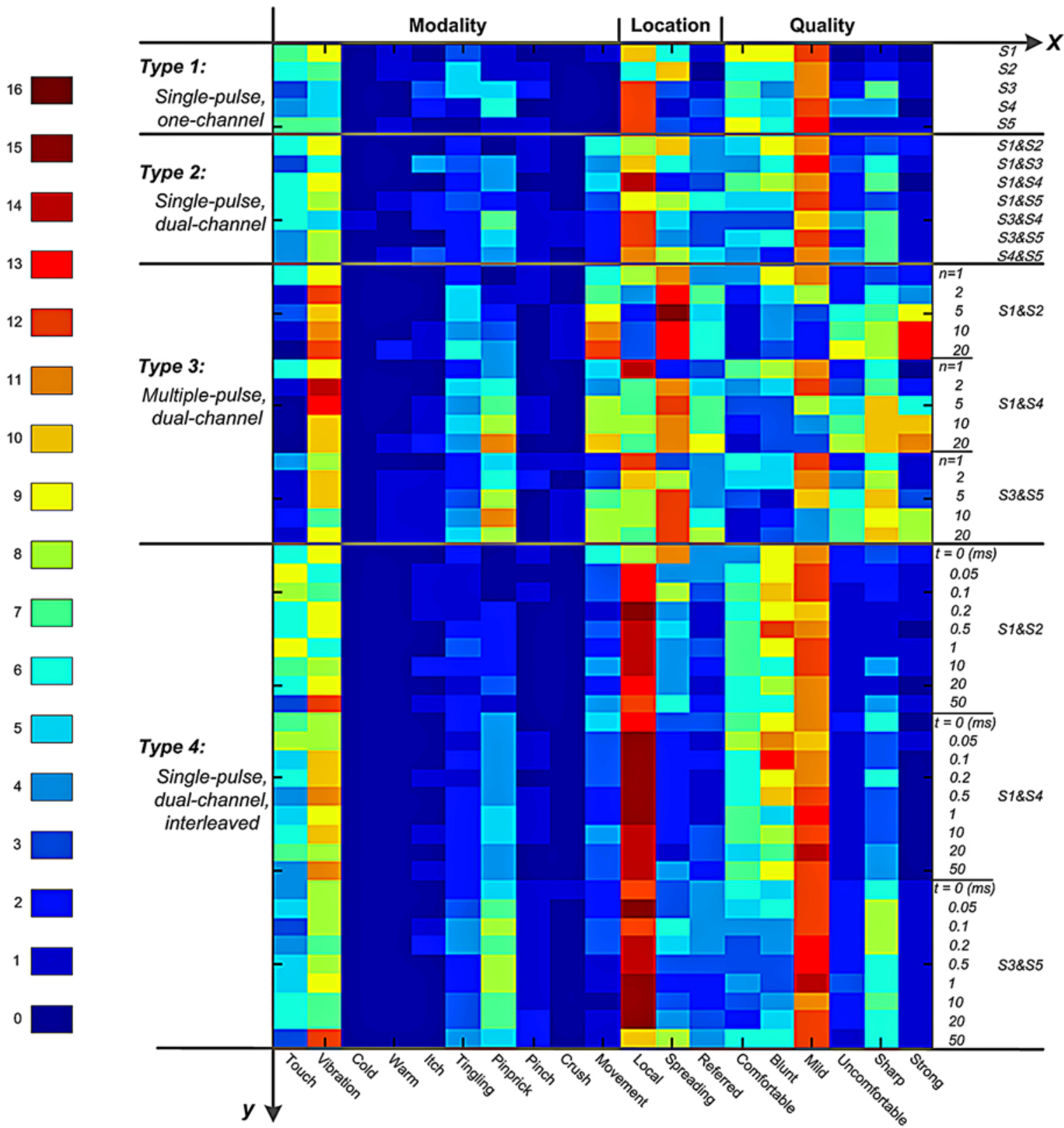

Figure 2.

Heat map representation of sensation modality, location, and quality collected from 16 subjects. Colors encode number of subjects reporting specific sensation ( $x$-axis). Warmer color (i.e., red) indicates that more subjects chose the word, while cooler color (i.e., blue) indicates fewer subjects. The four blocks each correspond to one of four types of stimulation ( $y$-axis, left) comprising 48 stimulation parameter combinations in total ( $y$-axis, right).

stimulation (type 4) induced "local" sensation more often. With respect to the sensation quality, "sharp," "uncomfortable," and "strong" qualities appeared to be more associated with the multiple-pulse stimulation (type 3). The "blunt" quality appeared more associated with the interleaved stimulation (type 4).

\section{Effects of Stimulation Site in Single-Channel Stimula- tion (Type 1)}

The percentage of subjects who reported "touch," "vibration," "itch," "tingling," or "pinprick" sensation modalities in the single-pulse, single-channel stimulation (type 1) at the five sites is shown in Figure 3(a). Sensation 
(a)

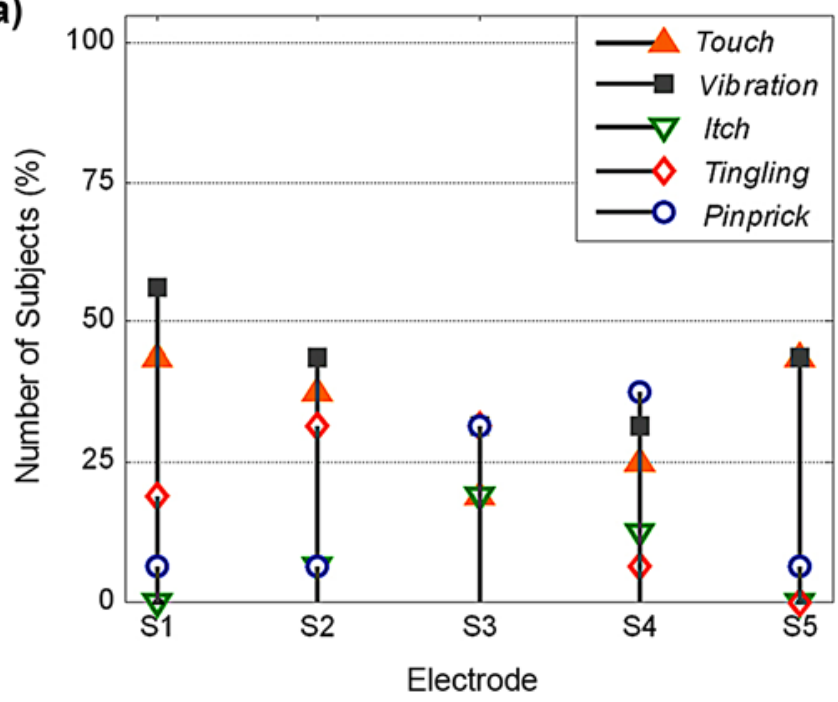

(b)

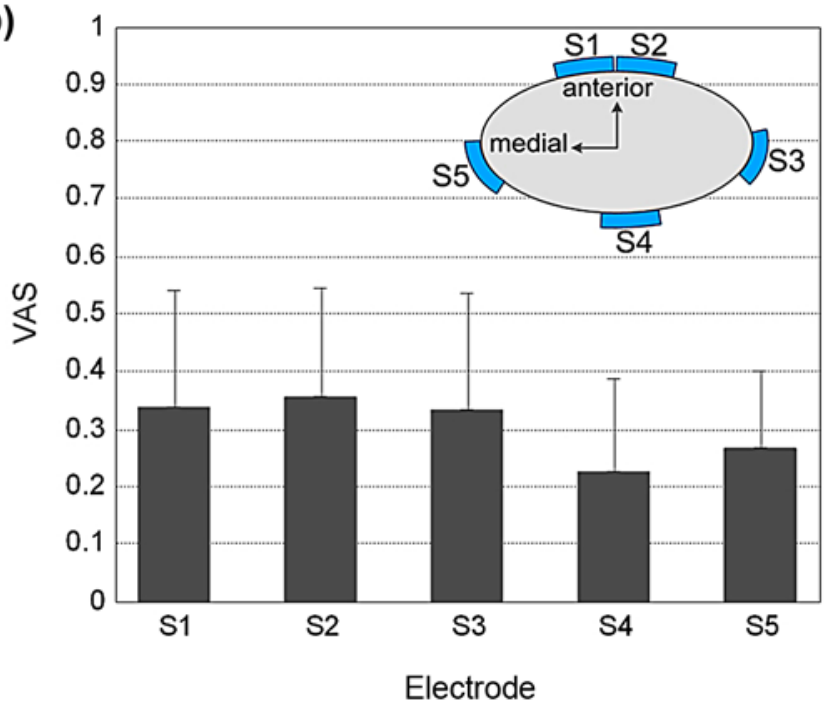

Figure 3.

Sensory modalities and visual analog scale (VAS) scores measured in single-pulse, single-channel stimulation (type 1). (a) Percentage of subjects reporting "touch," "vibration," "itch," "tingling," or "pinprick" modalities. (b) Mean and standard deviation of normalized VAS scores $(n=16)$.

modalities reported by less than three subjects are not shown (i.e., "cold," "warm," "pinch,” "crush,” "movement"). One can observe that "touch" and "vibration" were more frequently evoked at S1 and S5, "tingling" at S2 and S3, and "pinprick" and "itch" at S3 and S4. The results indicated that the stimulation site had a significant effect on evoking "touch" $(p<0.01)$, "vibration" ( $p<$ $0.01)$, "tingling” ( $p<0.05)$, and "pinprick" sensations $(p<0.05)$.

Figure 3(b) plots the normalized VAS scores when each of the five sites was stimulated. The sensation magnitude evoked at S4 (located on the dorsal side of the forearm) appeared to be the lowest. A significant effect of the stimulation site on the evoked sensation magnitude was found $(p<0.05)$.

From the heat map in Figure 2, one can observe that stimulation of S2 produced the "spreading" perception most frequently (63\%), while stimulation of S3 produced the "sharp" quality most frequently (44\%) and stimulation of S4 evoked the "uncomfortable" quality most frequently (25\%). The statistical results indicated a significant effect of stimulation site on the likelihood of evoking a "spreading” $(p<0.01)$, “sharp" $(p<0.05)$, or "uncomfortable” perception $(p<0.05)$.

\section{Effects of Single-Channel Versus Dual-Channel Stimu- lation (Type 1 vs Type 2)}

The effect of adding a second stimulating channel on evoked sensations was evaluated by comparing singlechannel stimulation at S1 with dual-channel stimulation where S1 was combined with the other four sites, i.e., S1\&S2, S1\&S3, S1\&S4, and S1\&S5. The frequency of reporting "movement" perception was found to be significantly increased $(p<0.05)$ when S1 was combined with S2 and S4 (Figure 4(a)). The VAS scores (Figure 4(b)) have also been compared. The results indicate that only inclusion of S2, which was located adjacent to S1, significantly increased the sensation magnitude $(p<0.05)$. No significant effects of adding a second channel on the location and quality of the evoked sensations were found.

\section{Effects of Pulse Number (Type 3)}

The upper row in Figure 5 shows the percentage of subjects reporting "touch,” "vibration,” "tingling," "pinprick," and "movement" modalities with increasing number of pulses ( $n=1,2,5,10,20)$ when the stimulation was applied to S1\&S2, S1\&S4, S3\&S5. "Cold," "warm," "itch," "pinch," and "crush" modalities were not included, because they either were not influenced by the 
(a)

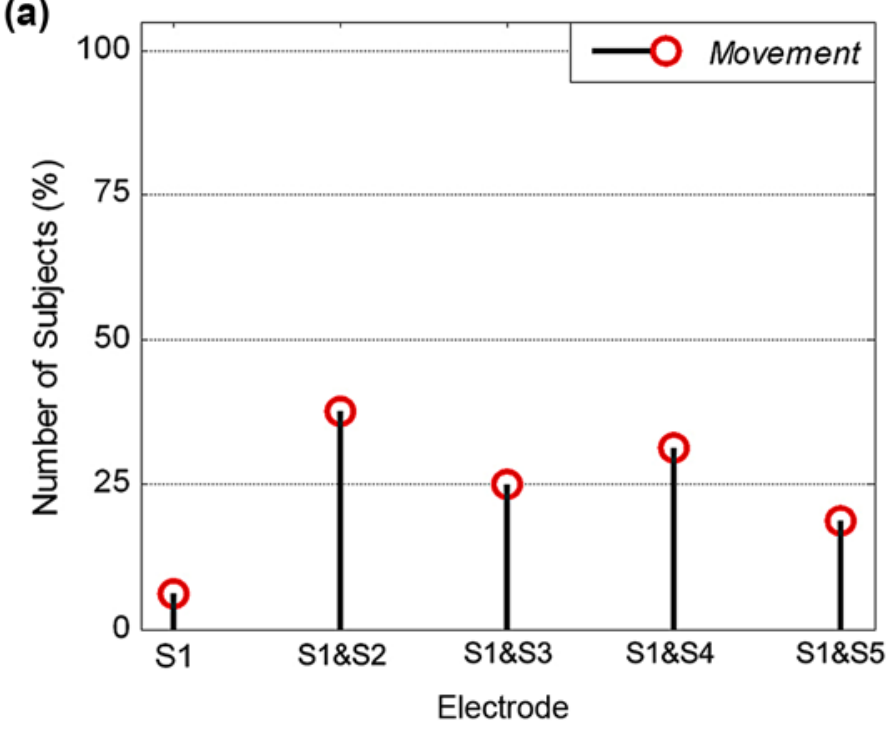

(b)

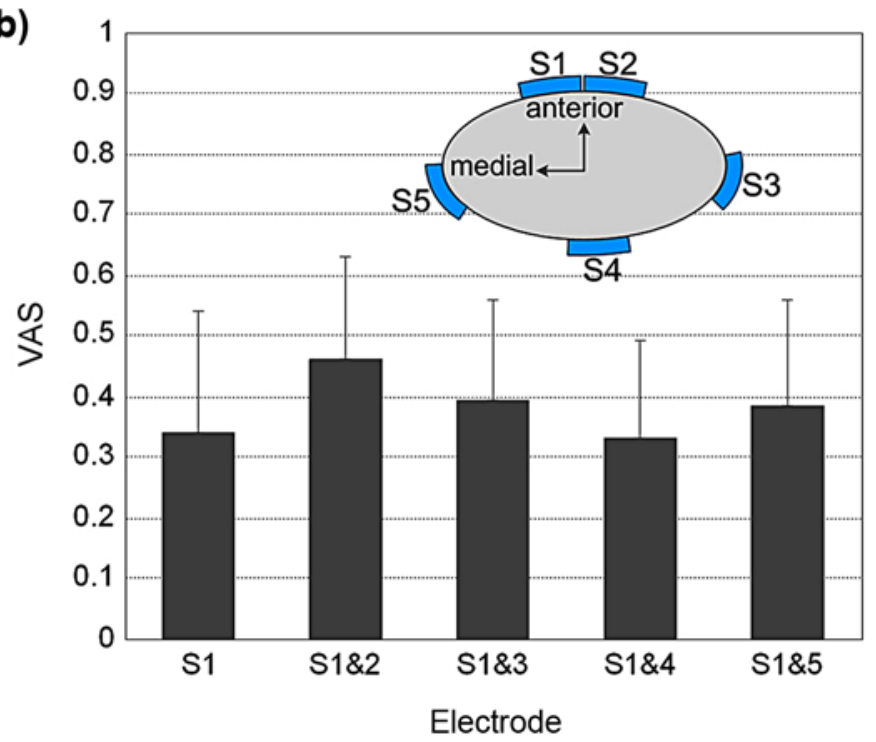

Figure 4.

Comparison between single-channel stimulation of S1 (type 1) and dual-channel stimulation of S1 combined with other four electrodes (type 2). (a) Percentage of subjects reporting "movement." (b) Mean and standard deviation of normalized visual analog scale (VAS) scores $(n=16)$.

pulse number or were reported by only one or two subjects.

One can observe that an increase in the number of pulses decreased the likelihood of inducing the "touch" sensation modality but increased the likelihood of eliciting "tingling," "pinprick," and "movement" perceptions irrespective of the stimulation site. The results indicated that the number of pulses had a significant effect on the "touch," "tingling," "pinprick," and "movement" modalities ( $p<0.01$ in all cases) but no significant effect on "vibration." The number of pulses also had a significant effect on the "spreading" perception $(p<0.01)$ and all six sensation qualities (i.e., $p<0.01$ for "comfortable," "blunt," "mild," "uncomfortable," "sharp," and "intense”).

When the three electrode pairs are compared, relatively fewer subjects perceived "vibration" at S3\&S5. Least frequent "pinprick" and most frequent "movement" both occurred at S1\&S2. The chance of perceiving "tingling” appeared to be lower at S3\&S5 than S1\&S2 and S1\&S4.

Figure 6(a) shows the VAS scores in the multiplepulse stimulation (type 3). The magnitude of evoked sensations increased significantly with higher number of pulses ( $p<0.01$ for all three electrode pairs). With use of regression analysis, a logarithmic relationship was found between the pulse number $n$ and the sensation magnitude
$M: M=0.454+0.138 \cdot \log (n), M=0.311+0.145 \cdot \log (n)$, and $M=0.307+0.145 \cdot \log (n)$ in the stimulation of S1\&S2, S1\&S4, and S3\&S5, respectively.

\section{Effects of Interleaved Time Between Two Channels (Type 4)}

The lower row in Figure 5 shows the percentages of subjects reporting "touch," "vibration," "tingling," "pinprick," and "movement" modalities with increasing interleaved time ( $t=0,0.05,0.1,0.2,0.5,1,5,10,50 \mathrm{~ms})$ when the stimulation was applied to S1\&S2, S1\&S4, S3\&S5. In stimulation of S1\&S2, the frequency of reporting the "movement" perception decreased with increasing interleaved time and became relatively consistent above $0.2 \mathrm{~ms}$. We speculated that an interleaved time shorter than $0.2 \mathrm{~ms}$ (i.e., pulse duration) may have resulted in a temporal summation of stimulus fields, which led to the "movement" perception. When the interleaved time exceeded $0.2 \mathrm{~ms}$, the dual-channel interleaved stimuli were equivalent to a single-channel stimulus with two pulses, which in this case did not reach the threshold of the "movement" perception. When the three electrode pairs were compared, the "touch" modality appeared to be elicited most frequently at S1\&S2. The "pinprick" modality was reported most frequently at S3\&S5. 
S1\&S2
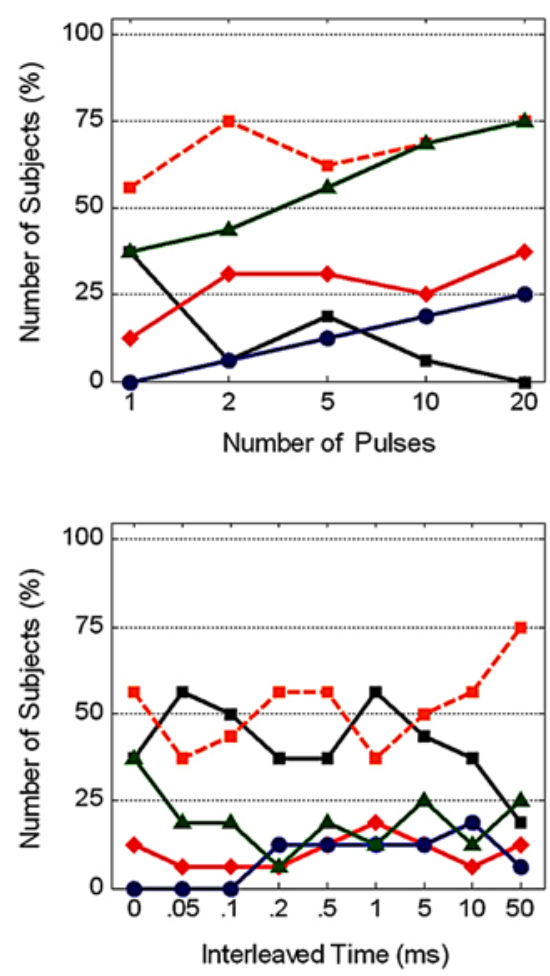

S1\&S4
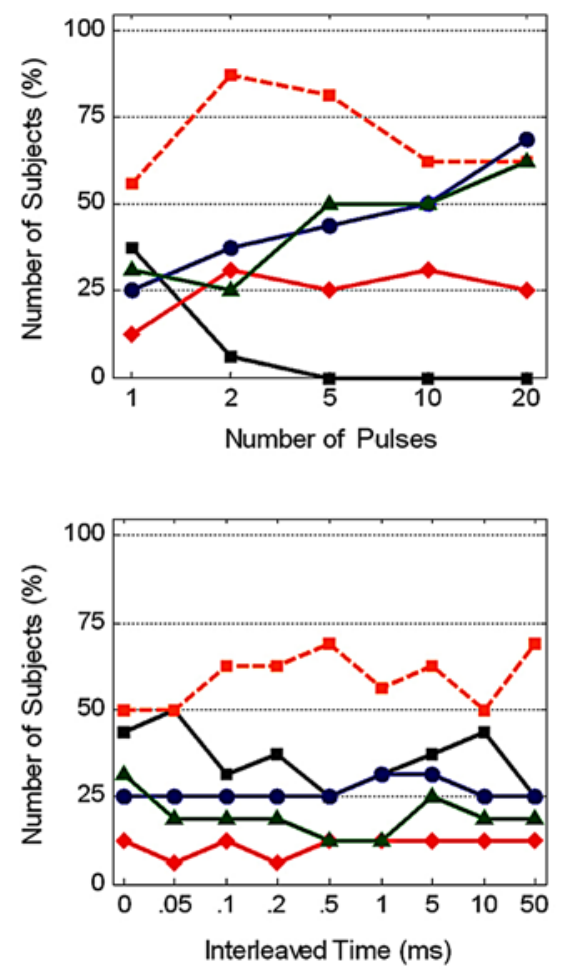

S3\&S5
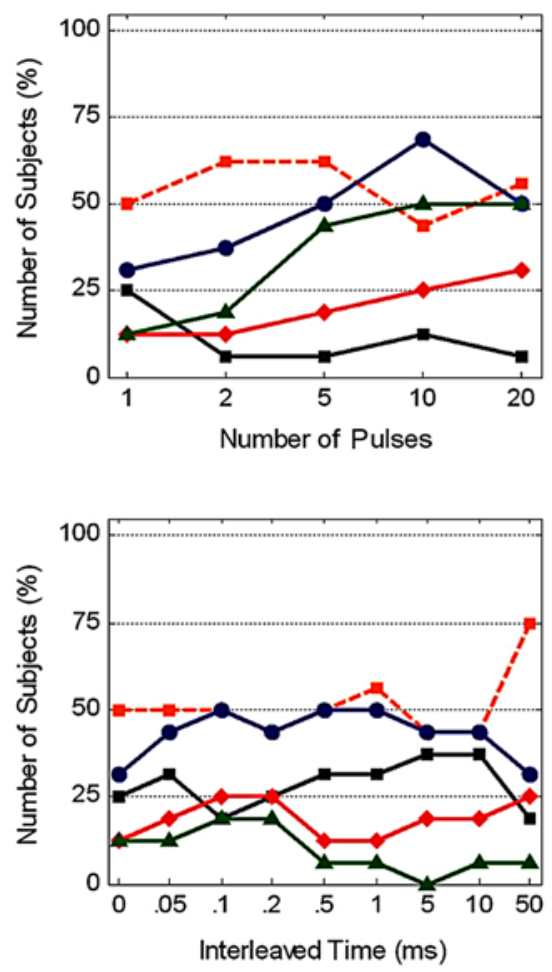

$\longrightarrow$ Touch --av-- Vibration $\longrightarrow$ Tingling $\longrightarrow$ Pinprick Movement

Figure 5.

Percentage of subjects reporting on evoked sensation modalities with series of increasing number of pulses in multiple-pulse, dualchannel stimulation (type 3, upper row) and increasing interleaved time in single-pulse, dual-channel interleaved stimulation (type 4, lower row) when stimulation applied to S1\&S2 (left), S1\&S4 (middle), and S3\&S5 (right).

Compared with the dual-channel simultaneous stimulation, the interleaved stimulation significantly increased the chance of producing "local" perception $(p<0.01)$ at S1\&S2. With regard to the sensation quality, an interleaved time shorter than 1 ms showed a significant effect on "blunt" feeling $(p<0.05)$ in all three electrode pair combinations; i.e., a longer interleaved time produced the "blunt" sensation more frequently. However, the results indicated no significant effect of the interleaved time on the VAS score (Figure 6(b)).

\section{DISCUSSION}

\section{On Assessment of Perceived Sensations}

The predefined words for the subjects describing the modality of perceived sensations were selected according to three sensation modalities of human skin: tactile (touch, vibration), thermal (cold, warm), and pain (pinprick, pinch, and crush) [20-21]. In addition, tingling, itch and movement were observed in the pilot experiment and, thus, also included in the answer list. Different sets of words have been used to describe electrical stimulation elicited sensations in earlier work (e.g., [2,6,17-18]). A subset of words was selected not only to eliminate redundancy but also to avoid attention and/or distraction arising from a long answer list, which may introduce bias during psychophysical tests [22]. In most cases, the subjects had no problem finding the words that described their perception. Only one subject reported "shock," which was absent from the list. As such, the word list used in the present work was believed to cover the majority of possible cutaneous sensory modalities. 
(a)

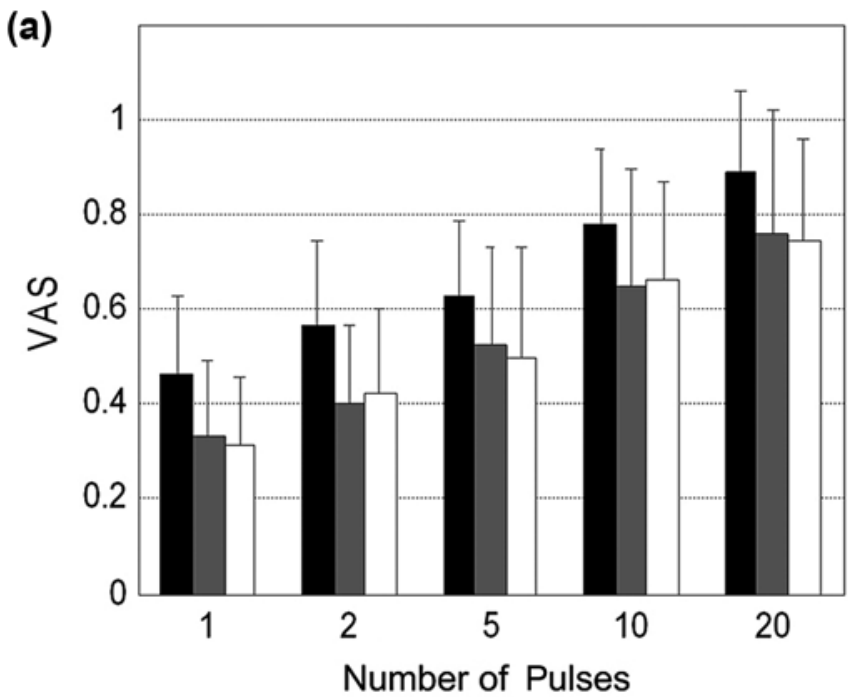

(b)

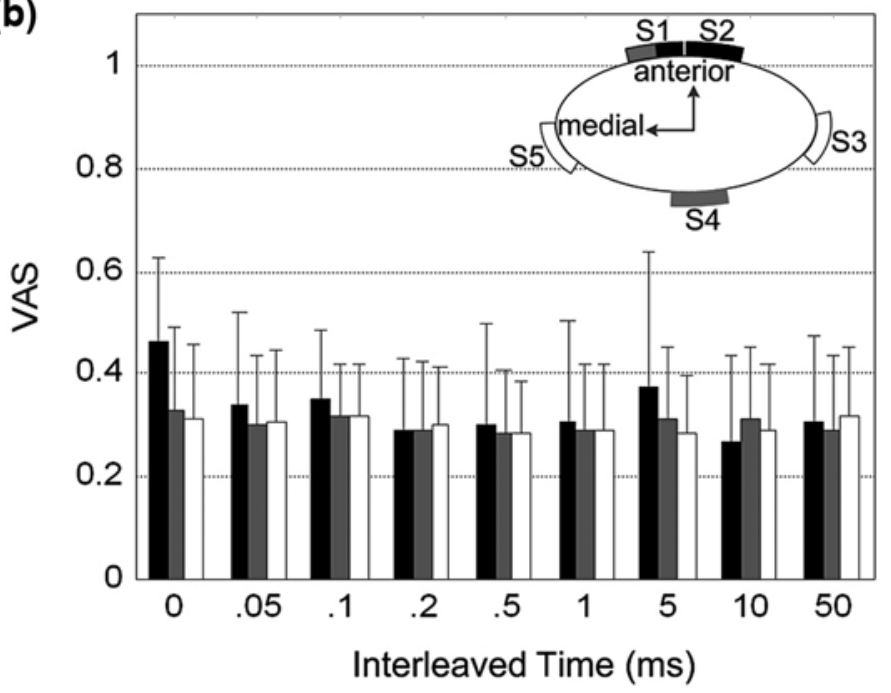

Figure 6.

Normalized visual analog scale (VAS) scores as function of (a) number of pulses in dual-channel, simultaneous stimulation (type 3) and (b) interleaved time in single-pulse, dual-channel stimulation (type 4). Each bar represents mean and standard deviation ( $n=16$ ). Note: In inset schematic of electrode placement, colors indicating three electrode pair combinations correspond to colors of bars.

The subjects were generally prone to choose the words "mild," "intense," "sharp," or "blunt” rather than "comfortable" or "uncomfortable" to describe the quality of a sensation they perceived. Most subjects reported that neither "comfortable" nor "uncomfortable" could describe the quality of the artificially created sensations in the experiment. This may suggest that "comfortable" is not an appropriate word for evaluating the acceptance of artificial sensory feedback induced by electrocutaneous stimulation.

The order of presenting stimuli to the subjects within each stimulation type was the same. Note that maintaining the same order among the subjects might have affected the results by making the measurement results dependent on one other [23]. It is not possible with the current data to determine whether an order effect existed in the present experiment. Presentation of the stimuli in a different or random order may eliminate any possible bias. However, running a set of fully randomized experiments is not feasible.

\section{On Effects of Stimulation Site on Evoked Sensations}

"Touch" or "vibration" sensations could not be induced with equal chances at the five sites in the forearm. When the ventral side of the forearm skin was stimulated (S1), all 16 subjects perceived "touch" or "vibration," whereas 14 out of $16(88 \%)$ reported the same when the ulnar side was stimulated (S5). This number was reduced to 8 (50\%) and 9 (56\%) during stimulation of the dorsal side (S4) and the radial side (S3), respectively. Conversely, the "pinprick" sensation was reported more frequently on the dorsal and radial side than the ventral and ulnar side. Since Pacinian corpuscles and Merkel disk receptors respond to vibration and light touch (or pressure) [24], this result may suggest that the distribution of these two kinds of receptors is denser on the ventral forearm. In practical applications, one may consider "touch" and "vibration" to be highly useful. This result also implies that the ventral and ulnar side of the forearm may be a more suitable target to receive artificial sensory feedback by electrocutaneous stimulation.

A "spreading" sensation was more frequently reported on the ventral side of the forearm. The speculation is that the afferent neurons in a bigger skin area may have been activated or that the afferents whose receptors are located distally outside the range of stimulation were activated. When stimulation was applied to the ventral side of the forearm (S1 and S2), 6 (38\%) and 10 subjects (63\%), respectively, perceived the sensation in a spread area, while only 2 (13\%) subjects reported "spreading” perception on the dorsal side (S4). This difference may suggest that skin conductivity on the ventral side is higher than on the dorsal side of the forearm, and therefore, the current 
field propagated in a larger area on the ventral side. The difference may also be explained by different innervation patterns of skin receptors and/or proximity to the median nerve [25-26].

\section{On Effects of Pulse Number on Evoked Sensations}

In a majority of the single-pulse stimulations under investigation, the evoked sensations were localized on the skin under the stimulating electrodes. When the number of pulses increased, however, the evoked sensation typically spread distally in the forearm. The intensity gradient of the sensation was in the proximal to distal direction. The extent of the spread was positively related to the number of pulses; i.e., the higher number of pulses caused a larger spread. This may be explained by the stimulus current field activating a progressively larger set of afferent neurons with increasing charge injection [27-28].

An increase in the number of pulses remarkably increased the magnitude of perceived sensation. A logarithmic relationship was found between the pulse number and the sensation magnitude. In the previous study on the effects of pulse number on PT, an inverse relationship was found between PT and the pulse number: PT $=\mathrm{c}+\mathrm{d} / \mathrm{n}$ [29]. This may imply that the PT is negatively related to the sensation magnitude in a nonlinear fashion.

When the number of pulses was increased, movement was observed. The observed movement was finger or wrist flexion. The extent of flexion appeared to be positively correlated with the number of pulses in the stimuli.

\section{On Movement Perception}

The perception of "movement" does not necessarily imply that there was muscle activation; likewise, the report of other percepts does not necessarily imply that there was no motor activity present. The observation that hand/wrist flexion increased with multiple pulses may indicate a summation of sustained muscle contractions. The first pulse may already have induced a twitch contraction. A second pulse at the rate of 20 pulses per second, applied before the muscle has completely relaxed, induces another contraction that adds to the first. The summation of the contractions was greater than that of a single twitch [30]. As such, muscle activation might have already occurred in single-pulse dual-channel stimulation, but it was visible only with multiple pulses.

In this study, the muscle activity and joint kinematics were not measured with electromyography or video recordings. The word "movement" included in the psychophysical questionnaire should only indicate a subjective evaluation of movement perception. However, future investigation of how muscle activity is related to movement perception may be valuable.

\section{On Use of Electrocutaneous Stimulation in Practical Applications}

The results of the present work can be useful for sensory substitution and augmentation in stimulation of healthy skin and also have implications in the sensory feedback for hand prostheses and sensory discriminationbased treatment for PLP.

People with upper-limb amputation strongly desire sensory feedback in a hand prosthetic device [31]. With the use of multiple surface electrodes, the location of evoked sensations can be controlled through modulation of the stimulated site. A given electrode or electrode pair may be used as a channel to convey sensory information to one finger, and the user can relate the perception from different channels to different fingers [32].

The number of pulses and stimulation site significantly affected the evoked sensations. Hence, the stimulation paradigms based on modulation of these two parameters may be used in a sensory discrimination training program for PLP treatments [12-13].

Note that the results may not directly relate to the treatment of patients with amputation when stimulating the damaged limb, because their perceptual measures may differ from those in nondisabled subjects. Hence, it will be of interest to see whether similar results can be obtained with a population of persons with amputation in future work. As a limitation, only a selected set of stimulation profiles and locations were tested in the present study.

\section{CONCLUSIONS}

In this work, the sensations evoked by a variety of single- and dual-channel surface stimulation patterns on the forearm skin of nondisabled subjects were evaluated. Based on the results, the following conclusions and recommendations have been made. First, the stimulation site significantly affected the evoked sensations. Stimulation of the ventral and ulnar aspect of the forearm showed more consistency in the evoked sensation modality and a better chance of a "touch" or "vibration" perception. Also, the number of pulses was found to significantly affect the 
evoked sensations, which may imply that modulation of the pulse number is an effective way to convey information in sensory communication or sensory discrimination. In addition, the sensation magnitude appeared to be easier to modulate than modality, location, and quality. These findings suggest that appropriate selection of stimulation patterns is important to artificially induce reliable sensations in an electrocutaneous sensory feedback system of prosthetic devices or in a sensory discrimination program for PLP relief.

\section{ACKNOWLEDGMENTS}

\section{Author Contributions:}

Fundamental concept of study: W. Jensen, K. Yoshida, B. Geng. Experimental protocol design: B. Geng, W. Jensen, K. Yoshida, L. Petrini.

Acquisition of data and statistical analysis: B. Geng. Interpretation of results: B. Geng, W. Jensen, K. Yoshida, L. Petrini. Preparation of manuscript: B. Geng, W. Jensen, K. Yoshida, L. Petrini. Financial Disclosures: The authors have declared that no competing interests exist.

Funding/Support: This material was based on work supported by the Transverse, Intrafascicular Multichannel Electrode system project (grant CP-FP-INFSO 224012/TIME from the European Union).

Institutional Review: The ethics committee for Region Nordjylland, Denmark, approved this study (ref: VN-20090009), and the authors obtained informed consent from all participants.

Participant Follow-Up: The authors do not plan to inform participants of the publication of this study. However, participants have been encouraged to follow up the publication of this study by contacting the authors.

\section{REFERENCES}

1. Herberts P, Körner L. Ideas on sensory feedback in hand prostheses. Prosthet Orthot Int. 1979;3(3):157-62. PMID: 523298

2. Pfeiffer EA. Electrical stimulation of sensory nerves with skin electrodes for research, diagnosis, communication and behavioral conditioning: A survey. Med Biol Eng. 1968; 6(6):637-51. PMID: 4891827

http://dx.doi.org/10.1007/BF02474726

3. Kaczmarek KA, Webster JG, Bach-y-Rita P, Tompkins WJ. Electrotactile and vibrotactile displays for sensory substitution systems. IEEE Trans Biomed Eng. 1991;38(1):1-16. PMID: 2026426 http://dx.doi.org/10.1109/10.68204

4. Van Doren CL, Riso RR, Milchus K. Sensory feedback for enhancing upper extremity neuromuscular prostheses. Neu- rorehabil Neural Repair. 1991;5(1-2):63-74.

http://dx.doi.org/10.1177/136140969100500107

5. Riso RR. Strategies for providing upper extremity amputees with tactile and hand position feedback-Moving closer to the bionic arm. Technol Health Care. 1999;7(6): 401-9. PMID: 10665673

6. Szeto AY, Saunders FA. Electrocutaneous stimulation for sensory communication in rehabilitation engineering. IEEE Trans Biomed Eng. 1982;29(4):300-8. PMID: 7068167 http://dx.doi.org/10.1109/TBME.1982.324948

7. Beeker TW, During J, Den Hertog A. Artificial touch in a hand-prosthesis. Med Biol Eng. 1967;5(1):47-49.

PMID: 6037625 http://dx.doi.org/10.1007/BF02478841

8. Schmidl $\mathrm{H}$. The importance of information feedback in prostheses for the upper limbs. Prosthet Orthot Int. 1977; 1(1):21-24. PMID: 615983

9. Shannon GF. A myoelectrically-controlled prosthesis with sensory feedback. Med Biol Eng Comput. 1979;17(1):73-80. PMID: 312386 http://dx.doi.org/10.1007/BF02440956

10. Prior RE, Lyman J, Case PA, Scott CM. Supplemental sensory feedback for the VA/NU myoelectric hand: Background and preliminary designs. Bull Prosthet Res. 1976; 10(26):170-91. PMID: 1030327

11. Scott RN, Brittain RH, Caldwell RR, Cameron AB, Dunfield VA. Sensory-feedback system compatible with myoelectric control. Med Biol Eng Comput. 1980;18(1):65-69. PMID: 7382591 http://dx.doi.org/10.1007/BF02442481

12. Flor H, Denke C, Schaefer M, Grüsser S. Effect of sensory discrimination training on cortical reorganisation and phantom limb pain. Lancet. 2001;357(9270):1763-64. PMID: 11403816 http://dx.doi.org/10.1016/S0140-6736(00)04890-X

13. Moseley GL, Zalucki NM, Wiech K. Tactile discrimination, but not tactile stimulation alone, reduces chronic limb pain. Pain. 2008;137(3):600-8. PMID: 18054437 http://dx.doi.org/10.1016/j.pain.2007.10.021

14. Szeto AY, Lyman J, Prior RE. Electrocutaneous pulse rate and pulse width psychometric functions for sensory communications. Hum Factors. 1979;21(2):241-49.

PMID: 489023

15. Szeto AY. Relationship between pulse rate and pulse width for a constant-intensity level of electrocutaneous stimulation. Ann Biomed Eng. 1985;13(5):373-83.

PMID: 4073624

http://dx.doi.org/10.1007/BF02407767

16. Van Doren CL. Contours of equal perceived amplitude and equal perceived frequency for electrocutaneous stimuli. Percept Psychophys. 1997;59(4):613-22. PMID: 9158335 http://dx.doi.org/10.3758/BF03211869 
17. Sang CN, Max MB, Gracely RH. Stability and reliability of detection thresholds for human A-beta and A-delta sensory afferents determined by cutaneous electrical stimulation. J Pain Symptom Manage. 2003;25(1):64-73.

PMID: 12565190 http://dx.doi.org/10.1016/S0885-3924(02)00541-9

18. Anani AB, Ikeda K, Körner LM. Human ability to discriminate various parameters in afferent electrical nerve stimulation with particular reference to prostheses sensory feedback. Med Biol Eng Comput. 1977;15(4):363-73. PMID: 197328 http://dx.doi.org/10.1007/BF02457988

19. Schriesheim CA, Kopelman RE, Solomon E. The effect of grouped versus randomized questionnaire format on scale reliability and validity: A three-study investigation. Educ Psychol Meas. 1989;49(3):487-508. http://dx.doi.org/10.1177/001316448904900301

20. Burgess PR, Petit D, Warren RM. Cutaneous mechanoreceptors and nociceptors. In: Iggo A, editor. Handbook of sensory physiology, Vol II: Somatosensory system. New York (NY): Springer-Verlag; 1973. p. 28-78.

21. Hensel H. Cutaneous thermoreceptors. In: Iggo A, editor. Handbook of sensory physiology, Vol II: Somatosensory system. New York (NY): Springer-Verlag; 1973. p. 79-110.

22. Gescheider GA, editor. Psychophysics: The fundamentals. 3rd ed. Mahwah (NJ): Lawrence Erlbaum Associates; 1997.

23. Cohen PR. Empirical methods for artificial intelligence. Cambridge (MA): MIT Press; 1995.

24. Gardner EP, Martin JH, Jessell TM. The bodily senses. In: Kandel ER, Schwartz JH, Jessell TM, editors. Principles of neural science. 4th ed. New York (NY): McGraw-Hill; 2000. p. 432.

25. Vallbo AB, Olausson H, Wessberg J, Kakuda N. Receptive field characteristics of tactile units with myelinated afferents in hairy skin of human subjects. J Physiol. 1995; 483(Pt 3):783-95. PMID: 7776258

26. Wessberg J, Olausson H, Fernström KW, Vallbo AB. Receptive field properties of unmyelinated tactile afferents in the human skin. J Neurophysiol. 2003;89(3):1567-75. PMID: 12626628 http://dx.doi.org/10.1152/jn.00256.2002
27. Meier JH, Rutten WL, Zoutman AE, Boom HB, Bergveld P. Simulation of multipolar fiber selective neural stimulation using intrafascicular electrodes. IEEE Trans Biomed Eng. 1992;39(2):122-34. PMID: 1612615 http://dx.doi.org/10.1109/10.121643

28. Nannini N, Horch K. Muscle recruitment with intrafascicular electrodes. IEEE Trans Biomed Eng. 1991;38(8):769-76. PMID: 1937510 http://dx.doi.org/10.1109/10.83589

29. Geng B, Yoshida K, Jensen W. Impacts of selected stimulation patterns on the perception threshold in electrocutaneous stimulation. J Neuroeng Rehabil. 2011;8:9.

PMID: 21306616 http://dx.doi.org/10.1186/1743-0003-8-9

30. Loeb GE, Ghez C. The motor unit and muscle action. In: Kandel ER, Schwartz JH, Jessell TM, editors. Principles of neural science. 4th ed. New York (NY): McGraw-Hill; 2000. p. 674-86.

31. Atkins DJ, Heard DC, Donovan WH. Epidemiologic overview of individuals with upper-limb loss and their reported research priorities. J Prosthet Orthot. 1996;8(1):2-11. http://dx.doi.org/10.1097/00008526-199600810-00003

32. Dhillon GS, Krüger TB, Sandhu JS, Horch KW. Effects of short-term training on sensory and motor function in severed nerves of long-term human amputees. J Neurophysiol. 2005;93(5):2625-33. PMID: 15846000 http://dx.doi.org/10.1152/jn.00937.2004

Submitted for publication September 20, 2010. Accepted in revised form June 20, 2011.

This article and any supplementary material should be cited as follows:

Geng B, Yoshida K, Petrini L, Jensen W. Evaluation of sensation evoked by electrocutaneous stimulation on forearm in nondisabled subjects. J Rehabil Res Dev. 2012; 49(2):297-308.

http://dx.doi.org/10.1682/JRRD.2010.09.0187

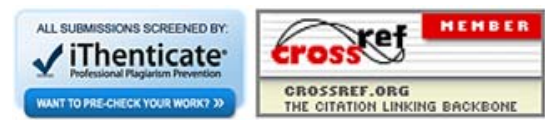

